\title{
LEIBNIZ ALGEBRAS, HAVING A DENSE FAMILY OF IDEALS
}

\author{
SEMKO N.N., SKASKIV L.V., YAROVAYA O.A. \\ We say that a Leibniz algebra $L$ has a dense family of ideals, if for every pair of subalgebras $A$, \\ $B$ of $L$ such that $A \leqslant B$ and $A$ is not maximal in $B$ there exists an ideal $S$ such that $A \leqslant S \leqslant B$. We \\ study the Leibniz algebras, having a dense family of ideals. \\ Key words and phrases: Leibniz algebra, Lie algebra, ideal, dense family, nilpotent Leibniz algebra.
}

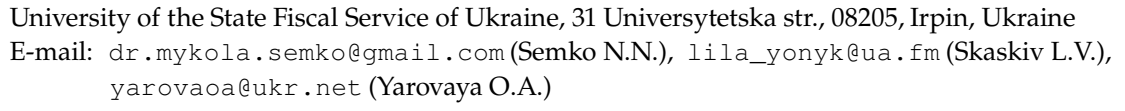

\section{INTRODUCTION}

Let $L$ be an algebra over a field $F$ with the binary operations + and $[\cdot, \cdot]$. Then $L$ is called a Leibniz algebra (more precisely a left Leibniz algebra), if it satisfies the Leibniz identity

$$
[a,[b, c]]=[[a, b], c]+[b,[a, c]]
$$

for all $a, b, c \in L$.

If $L$ is a Lie algebra, then $L$ is a Leibniz algebra. Conversely, if $L$ is a Leibniz algebra such that $[a, a]=0$ for each element $a \in L$, then $L$ is a Lie algebra. Therefore, Lie algebras can be characterized as the Leibniz algebras in which $[a, a]=0$ for every element $a \in L$.

Leibniz algebras appeared first in the papers of A. Blokh [2], in which he called them the $D$-algebras. However, in that time these works were not in demand, and they have not been properly developed. Only after two decades, a real interest to Leibniz algebras arose. It is happened thanks to the work of J.-L. Loday [16], who "rediscovered" these algebras and used the term Leibniz algebras.

One approach to the study of Leibniz algebras, which proved to be quite effective especially for infinite dimensional Leibniz algebras, is to consider of Leibniz algebras, all whose subalgebras have some fixed natural properties. This approach has been very effective for Lie algebras, while in Leibniz algebras it is starting to be used only recently. Thus in [4] the Leibniz algebras whose subalgebras are Lie algebras and Leibniz algebras whose subalgebras are abelian were studied.

In the paper [13] the Leibniz algebras whose subalgebras are ideals were studied. In the paper [15] the Leibniz algebras whose subideals are ideals were studied. More detailed information about this approach to the study of Leibniz algebras can be found in the survey papers $[5,6]$ and [14]. In this paper we start to study the Leibniz algebras, having very big family of ideals. 
Let $L$ be a Leibniz algebra over a field $F$ and $\mathfrak{S}$ be some family of subalgebras of $L$. We say that a family $\mathfrak{S}$ is dense in $L$, if for every pair of subalgebras $A, B$ of $L$ such that $A \leqslant B$ and $A$ is not maximal in $B$ there exists a subspace $S \in \mathfrak{S}$ such that $A \leqslant S \leqslant B$ (of course, $S$ can be coincides with $A$ or $B$ ).

The origins of this concept are in group theory. There is a whole array of articles devoted to the study of groups, having different natural families of subgroups (see [3,7-10,17-23]). This topic is not limited to the framework of the classical theory of groups. For example, in [24] the infinite dimensional linear groups having a dense family of subgroups of finite central dimension were considered.

One of most important family of $L$ is the family of ideals of $L$. Therefore, one of the first natural question is the study of Leibniz algebras, having a dense family of ideals.

The definition shows that Leibniz algebra, having a dense family of ideals, must have a very extensive system of ideals and, therefore, it is very close to Leibniz algebras, whose subalgebras are ideals. However, it should immediately be noted that in such algebras not all subalgebras are necessarily ideals. For example, every Leibniz algebra of dimension 3 clearly has a dense family of ideals.

Consider another example. Let $L$ be a cyclic nilpotent Leibniz algebra of dimension 4 . Then $L$ has a basis $\left\{a_{1}, a_{2}, a_{3}, a_{4}\right\}$ such that

$$
\left[a_{1}, a_{1}\right]=a_{2},\left[a_{1}, a_{2}\right]=a_{3},\left[a_{1}, a_{3}\right]=a_{4}
$$

and

$$
\left[a_{j}, a_{k}\right]=0
$$

for all $j>1$. Put $K=F a_{2} \oplus F a_{3} \oplus F a_{4}$, then clearly $K$ is an abelian ideal of $L$. Let $S$ be an arbitrary non-zero subalgebra of $L$. If $K$ does not include $S$, then $S=L$. Assume that $K$ includes $S$. If $D$ is a subalgebra of $L$ such that $D$ includes $S$ and $S$ is not maximal in $D$, then for $D$ we have only two possibilities: $D=K$ or $D=L$. In both cases $D$ is an ideal of $L$. This means that $L$ has a dense family of ideals.

A Leibniz algebra which is not a Lie algebra has one specific ideal. Denote by $\operatorname{Leib}(L)$ the subspace generated by the elements $[a, a], a \in L$. It is possible to prove that $\operatorname{Leib}(L)$ is an ideal of $L$. Moreover, $L / \operatorname{Leib}(L)$ is a Lie algebra. Conversely, if $H$ is an ideal of $L$ such that $L / H$ is a Lie algebra, then Leib $(L) \leqslant H$. The ideal Leib $(L)$ is called the Leibniz kernel of $L$.

As usual, a Leibniz algebra $L$ is abelian, if $[x, y]=0$ for each $x, y \in L$.

Let $L$ be a Leibniz algebra over a field $F$. If $A, B$ are subspaces of $L$, then $[A, B]$ will denote a subspace generated by all elements $[a, b]$ where $a \in A, b \in B$. If $M$ is a non-empty subset of $L$, then $\langle M\rangle$ denote the subalgebra of $L$ generated by $M$.

\section{MAIN RESULTS}

Lemma 1. Let $L$ be a Leibniz algebra, having a dense family of ideals. Then every abelian subalgebra of $L$, having dimension 3 , is an ideal of $L$.

Proof. Let $A$ be an abelian subalgebra of $L$, having dimension 3, then $A=F a \oplus F b \oplus F c$. Since $A$ is abelian, every subspace $F a, F b, F c$ is a subalgebra of $A$. A subspace $F a$ has codimension 2 in $A$, so that $F a$ is not maximal in $A$. Then there exists an ideal $D_{a}$ of $L$ such that $F a \leqslant$ $D_{a} \leqslant A$. By the similar reasons there are the ideals $D_{b}, D_{c}$ of $L$ such that $F b \leqslant D_{b} \leqslant A$ 
and $F_{c} \leqslant D_{c} \leqslant A$. We note that the sum $D_{a}+D_{b}+D_{c}$ is an ideal of $L$. The inclusions $A=F a \oplus F b \oplus F c \leqslant D_{a}+D_{b}+D_{c} \leqslant A$ shows that $A$ is an ideal of $L$.

Lemma 2. Let $L$ be a Leibniz algebra, having a dense family of ideals, and let $A$ be an abelian subalgebra of $L$. If $\operatorname{dim}_{F}(A) \geqslant 4$, then every subalgebra of $A$ is an ideal of $L$.

Proof. Clearly it is sufficient to prove that every cyclic subalgebra of $A$ is an ideal of $L$. Since $A$ is abelian, every cyclic subalgebra of $A$ has dimension 1. Let $a$ be an arbitrary element of $A$. Since $\operatorname{dim}_{F}(A) \geqslant 4$, we can choose a subspace $B$ of $A$ such that $a \in B$ and $\operatorname{dim}_{F}(B)=4$. Choose in $B$ a basis $\{a, b, c, d\}$. Since $A$ is abelian, $B$ and each subspace of $B$ are subalgebras of $L$. Since $A$ is abelian, every subspace $F a, F b, F c, F d$ is a subalgebra of $A$. Put $B_{a, b, c}=F a \oplus F b \oplus F c$. A subspace $F a$ has codimension 2 in $B_{a, b, c}$, so that $F a$ is not maximal in $B_{a, b, c}$. Then there exists an ideal $D_{a, b, c}$ of $L$ such that $F a \leqslant D_{a, b, c} \leqslant B_{a, b, c}$. Put $B_{a, b, d}=F a \oplus F b \oplus F d, B_{a, c, d}=F a \oplus F c \oplus F d$. By the similar reasons there are the ideals $D_{a, b, d}$ and $D_{a, c, d}$ of $L$ such that $F a \leqslant D_{a, b, d} \leqslant B_{a, b, d}$ and $F a \leqslant D_{a, c, d} \leqslant B_{a, c, d}$. We note that the intersection $D_{a, b, c} \cap D_{a, b, d} \cap D_{a, c, d}$ is an ideal of $L$. We note also that

$$
B_{a, b, c} \cap B_{a, b, d} \cap B_{a, c, d}=(F a \oplus F b \oplus F c) \cap(F a \oplus F b \oplus F d) \cap(F a \oplus F c \oplus F d)=F a .
$$

Thus the inclusions

$$
F a \leqslant D_{a, b, c} \cap D_{a, b, d} \cap D_{a, c, d} \leqslant B_{a, b, c} \cap B_{a, b, d} \cap B_{a, c, d}=F a
$$

shows that $F a$ is an ideal of $L$.

Corollary 1. Let $L$ be a Leibniz algebra, having a dense family of ideals. If $L$ includes an abelian subalgebra $A \geqslant \operatorname{Leib}(L)$ such that $\operatorname{dim}_{F}(A) \geqslant 4$, then every subalgebra of $L$ is an ideal of $L$.

Proof. Since $A$ is abelian, Lemma 2 implies that each subalgebra of $A$ is an ideal of $L$.

Let $a$ be an arbitrary element of $L$. If $a \in A$, then by above noted a subalgebra $\langle a\rangle=F a$ is an ideal of $L$.

Suppose that $a \notin A$. An inclusion Leib $(L) \leqslant A$ implies that $L / A$ is a Lie algebra. Every cyclic subalgebra of a Lie algebra has dimension 1 , so that $\langle a+A\rangle=F(a+A)$. Further $[a, a]=$ $b \in A$. By above noted a subalgebra $\langle b\rangle=F b$ is an ideal of $L$. Then $[a, b],[b, a] \in\langle b\rangle$, so that $\langle a\rangle=F a \oplus F b$. If $b=0$, then $\langle a\rangle=F a$. In every case $\langle a\rangle \cap A=\langle b\rangle=F b$. Since $\operatorname{dim}_{F}(A) \geqslant 4$, we can choose a subspace $B$ of $A$ such that $b \in B$ and $\operatorname{dim}_{F}(B)=4$.

Since $B$ is an ideal of $L,\langle a, B\rangle=\langle a\rangle+B$. Choose in $B$ a basis $\{b, u, v, w\}$. Put $B_{u, v}=$ $\langle a\rangle \oplus F u \oplus F v$. A subalgebra $\langle a\rangle$ has codimension 2 in $B_{u, v}$, so that $\langle a\rangle$ is not maximal in $B_{u, v}$. Then there exists an ideal $D_{u, v}$ of $L$ such that $\langle a\rangle \leqslant D_{u, v} \leqslant B_{u, v}$. Put $B_{u, w}=\langle a\rangle \oplus F u \oplus F w$, $B_{v, w}=\langle a\rangle \oplus F v \oplus F w$. By the similar reasons there are the ideals $D_{u, w}$ and $D_{v, w}$ of $L$ such that $\langle a\rangle \leqslant D_{u, w} \leqslant B_{u, w}$ and $\langle a\rangle \leqslant D_{v, w} \leqslant B_{v, w}$. We note that the intersection $D_{u, v} \cap D_{u, w} \cap D_{v, w}$ is an ideal of $L$. We note also that

$$
B_{u, v} \cap B_{u, w} \cap B_{v, w}=(\langle a\rangle \oplus F u \oplus F v) \cap(\langle a\rangle \oplus F u \oplus F w) \cap(\langle a\rangle \oplus F v \oplus F w)=\langle a\rangle .
$$

Thus the inclusions

$$
\langle a\rangle \leqslant D_{u, v} \cap D_{u, w} \cap D_{v, w} \leqslant B_{u, v} \cap B_{u, w} \cap B_{v, w}=\langle a\rangle
$$

shows that $\langle a\rangle$ is an ideal of $L$. Hence every cyclic subalgebra of $L$ is an ideal. It follows that every subalgebra of $L$ is an ideal of $L$. 
Lemma 3. Let $L$ be a Lie algebra, having a dense family of ideals, and let $A$ be an abelian subalgebra of $L$. If $\operatorname{dim}_{F}(A) \geqslant 4$, then every subalgebra of $L$ is an ideal of $L$. In particular, $L$ is abelian.

Proof. By Lemma 2 every subalgebra of $A$ is an ideal of $L$. Choose in $A$ a basis $\left\{a_{1}, a_{2}, a_{3}, a_{4}\right\}$. Let $x$ be an arbitrary element of $L$. Since $L$ is a Lie algebra, $[x, x]=0$, so that $\langle x\rangle=F x$. Since every subalgebra $\left\langle a_{j}\right\rangle=F a_{j}, 1 \leqslant j \leqslant 4$, is an ideal, $A_{1,2}=\left\langle a_{1}, a_{2}, x\right\rangle=\left\langle a_{1}\right\rangle \oplus\left\langle a_{2}\right\rangle \oplus\langle x\rangle$. It follows that a subalgebra $\langle x\rangle$ is not maximal in $A_{1,2}$. Therefore $A_{1,2}$ includes an ideal $D_{1,2}$ such that $\langle x\rangle \leqslant D_{1,2} \leqslant A_{1,2}$. By the similar reasons a subalgebra $A_{3,4}=\left\langle a_{3}, a_{4}, x\right\rangle=\left\langle a_{3}\right\rangle \oplus\left\langle a_{4}\right\rangle \oplus$ $\langle x\rangle$ includes an ideal $D_{3,4}$ such that $\langle x\rangle \leqslant D_{3,4} \leqslant A_{3,4}$. We note that the intersection $D_{1,2} \cap D_{3,4}$ is an ideal of $L$. We note also that

$$
A_{1,2} \cap A_{3,4}=\left(\left\langle a_{1}\right\rangle \oplus\left\langle a_{2}\right\rangle \oplus\langle x\rangle\right) \cap\left(\left\langle a_{3}\right\rangle \oplus\left\langle a_{4}\right\rangle \oplus\langle x\rangle\right)=\langle x\rangle .
$$

Thus the inclusions

$$
\langle x\rangle \leqslant D_{1,2} \cap D_{3,4} \leqslant A_{1,2} \cap A_{3,4}=\langle x\rangle
$$

shows that a subalgebra $\langle x\rangle$ is an ideal of $L$. Hence every cyclic subalgebra of $L$ is an ideal. It follows that every subalgebra of $L$ is an ideal of $L$.

Theorem 1. Let $L$ be a Leibniz algebra, having a dense family of ideals. If $L$ includes an abelian subalgebra $A$, having dimension at least 4 , then every subalgebra of $L$ is an ideal of $L$.

Proof. Put $K=\operatorname{Leib}(L)$. We note that $K$ is abelian. If $\operatorname{dim}_{F}(K) \geqslant 4$, then using Corollary 1 we obtain that each subalgebra of $L$ is an ideal of $L$. Suppose now that $\operatorname{dim}_{F}(K)<4$. Lemma 2 implies that every subalgebra of $A$ (and $A$ itself) is an ideal of $L$. Then $A+K$ is a nilpotent ideal of $L$ [1, Lemma 1.5]. If $a \in A$, then using again Lemma 2 we obtain that $\langle a\rangle=F a$ is an ideal of $L$. In particular, $\langle a\rangle$ is an ideal of $A+K$. Then the intersection $\langle a\rangle \cap \zeta(A+K)$ is non-zero [14, Lemma 2.4]. On the other hand, $\operatorname{dim}_{F}(\langle a\rangle)=1$. This means that $\langle a\rangle \leqslant \zeta(A+K)$. Since it is true for every element $a \in A$, we obtain that $A \leqslant \zeta(A+K)$. Then $A+K$ is abelian. Since $\operatorname{dim}_{F}(A+K) \geqslant \operatorname{dim}_{F}(A) \geqslant 4$, an application of Corollary 1 implies that every subalgebra of $L$ is an ideal of $L$.

The left (respectively right) center $\zeta^{\text {left }}(L)$ (respectively $\zeta^{\text {right }}(L)$ ) of a Leibniz algebra $L$ is defined by the rule:

$$
\zeta^{\text {left }}(L)=\{x \in L \mid[x, y]=0 \text { for each } y \in L\}
$$

(respectively,

$$
\left.\zeta^{\text {right }}(L)=\{x \in L \mid[y, x]=0 \text { for each } y \in L\}\right) .
$$

It is not hard to prove that the left center of $L$ is an ideal, but it is not true for the right center. Moreover, Leib $(L) \leqslant \zeta^{l e f t}(L)$, so that $L / \zeta^{l e f t}(L)$ is a Lie algebra. The right center is a subalgebra of $L$, and in general, the left and right centers are different. Moreover, they even may have different dimensions. The example which have been constructed in [11] show this.

The center $\zeta(L)$ of $L$ is defined by the rule:

$$
\zeta(L)=\{x \in L \mid[x, y]=0=[y, x] \text { for each } y \in L\} .
$$

The center is an ideal of $L$. In particular, we can consider the factor-algebra $L / \zeta(L)$. 
Lemma 4. Let $L$ be a Leibniz algebra and $K$ be an ideal of $L$. Then the center of $K$ is an ideal of $L$.

Proof. Put $Z=\zeta(K)$. Choose the arbitrary elements $x \in L, y \in K, z \in Z$. We have

$$
[[x, z], y]=[x,[z, y]]-[z,[x, y]]
$$

and

$$
[[z, x], y]=[z,[x, y]]-[x,[z, y]]
$$

The choice the elements $z, y$ implies that $[z, y]=0$. Since $K$ is an ideal of $L,[x, y] \in K$, therefore $[z,[x, y]]=0$, so that $[[x, z], y]=[[z, x], y]=0$. Thus $Z$ is an ideal of $L$.

Let $L$ be a Leibniz algebra, $B, C$ are ideals of $L$ such that $B \leqslant C$. The factor $C / B$ is called $L$-central, if $C / B \leqslant \zeta(L / B)$. In other words, $[C, L],[L, C] \leqslant B$ or $A^{A n n} n_{L / B}(C / B)=L / B$. The factor $C / B$ is called L-eccentric, if $\operatorname{Ann}_{L / B}(C / B) \neq L / B$.

If $A$ is an ideal of a Leibniz algebra $L$, then we define the upper $L$-central series

$$
\langle 0\rangle=\zeta_{L, 0}(A) \leqslant \zeta_{L, 1}(A) \leqslant \zeta_{L, 2}(A) \leqslant \ldots \zeta_{L, \alpha}(A) \leqslant \zeta_{L, \alpha+1}(A) \leqslant \ldots \zeta_{L, \gamma}(A)=\zeta_{L, \infty}(A)
$$

of $A$ by the following rule: $\zeta_{L, 1}(A)=\zeta_{L}(A)=\operatorname{Ann}_{A}(L), \zeta_{L, \alpha+1}(A) / \zeta_{L, \alpha}(A)=\zeta_{L}\left(A / \zeta_{L, \alpha}(A)\right)$ for all $\alpha$, and $\zeta_{L}\left(A / \zeta_{L, \gamma}(A)\right)=\langle 0\rangle$. The last term $\zeta_{L, \infty}(A)$ of this series is called the upper $L$-hypercenter of $A$. By this definition, every term of the upper $L$-central series of $A$ is an ideal of $L$.

An ideal $C$ of $L$ is said to be $L$-hypereccentric, if it has an ascending series

$$
\langle 0\rangle=C_{0} \leqslant C_{1} \leqslant C_{2} \leqslant \ldots C_{\alpha} \leqslant C_{\alpha+1} \leqslant \ldots C_{\gamma}=C
$$

of ideals of $L$ such that each factor $C_{\alpha+1} / C_{\alpha}$ is $L$-eccentric and $L$-chief for every ordinal $\alpha$.

We say that the ideal $A$ of $L$ has the Z-decomposition if

$$
A=\zeta_{L, \infty}(A) \oplus \eta_{L, \infty}(A)
$$

where $\eta_{L, \infty}(A)$ is the maximal $L$-hypereccentric ideal of $A$. It is possible to prove that in this case $\eta_{L, \infty}(A)$ includes every $L$-hypereccentric ideal of $L$, in particular, this decomposition is unique.

Let $L$ be a Leibniz algebra over a field $F, M$ be non-empty subset of $L$ and $H$ be a subalgebra of L. Put

$$
\operatorname{Ann}_{H}^{\text {left }}(M)=\{a \in H \mid[a, M]=\langle 0\rangle\}
$$

and

$$
\operatorname{Ann}_{H}^{\text {right }}(M)=\{a \in H \mid[M, a]=\langle 0\rangle\} .
$$

The subset $\operatorname{Ann}_{H}^{\text {left }}(M)$ is called the left annihilator or the left centralizer of $M$ in $H$. The subset $\operatorname{Ann}_{H}^{\text {right }}(M)$ is called the right annihilator or the right centralizer of $M$ in $H$. The intersection

$$
\operatorname{Ann}_{H}(M)=\operatorname{Ann}_{H}^{\text {left }}(M) \cap \operatorname{Ann}_{H}^{\text {right }}(M)=\{a \in H \mid[a, M]=\langle 0\rangle=[M, a]\}
$$

is called the annihilator or the centralizer of $M$ in $H$.

It is not hard to see that all of these subsets are subalgebras of $L$. Moreover, it is possible to prove that if $M$ is a left ideal of $L$, then $\operatorname{Ann}_{L}^{\text {left }}(M)$ is an ideal of $L$. If $M$ is an ideal of $L$, then $\operatorname{Ann}_{L}(M)$ is an ideal of $L$. 
Lemma 5. Let $L$ be a Leibniz algebra, having a dense family of ideals. Suppose that a Leibniz kernel of $L$ has dimension at most 3 and $L /$ Leib $(L)$ includes an abelian subalgebra of dimension 4. Then $L$ is nilpotent and $L / \operatorname{Leib}(L)$ is abelian.

Proof. Put $K=\operatorname{Leib}(L)$. If the center of $L$ includes $K$, then clearly $L$ is nilpotent. Therefore suppose that the center of $L$ does not include $K$. Since the left center of $L$ includes $K$, $\operatorname{Ann}_{L}^{\text {right }}(K)=L$. It follows that $\operatorname{Ann}_{L}^{\text {left }}(K) \neq L$. Note that $L / \operatorname{Ann}_{L}^{\text {left }}(K)$ is isomorphic to some subalgebra of algebra of derivations of $K$ [11, Proposition 3.2]. It follows that this factoralgebra is finite dimensional and has dimension at most 9. By Lemma $3 \mathrm{~L} / \mathrm{K}$ is abelian.

Suppose that $\operatorname{Ann}_{L}^{\text {left }}(K)=\operatorname{Ann}_{L}(K) \neq L$. Let $x$ be an element of $L$ such that $x \notin \operatorname{Ann}_{L}(K)$. Then $\langle x, K\rangle$ is an ideal of $L$. Using Proposition 1.3 of [12] we obtain that $K$ has a direct decomposition $K=A \oplus B$, where

$$
A=\zeta_{\langle x\rangle, \infty}(A) \oplus \eta_{\langle x\rangle, \infty}(A) .
$$

Since $\langle x, K\rangle$ is an ideal of $L, A, B$ are also ideals of $L$. Moreover, by the choice of $B$ we obtain that $B=[x, B]$.

Suppose that a subspace $B$ is non-zero and consider a factor-algebra $L / A$. We have

$$
[x+A, K / A]=[x+A,(B+A) / A]=([x, B]+A) / A=(B+A) / A=K / A .
$$

Let $y$ be an arbitrary element of $L$. Since $L / K$ is abelian, $[x, y] \in K$, so that

$$
[x+A, y+A]=c+A \in K / A .
$$

An equality $K / A=(B+A) / A$ shows that $c+A=b+A$ for some $b \in B$. An equality $B=[x, B]$ implies that $b=[x, d]$ for some $d \in B$. Thus we have

$$
[x+A, y+A]=c+A=[x, d]+A=[x+A, d+A] .
$$

It follows that $[x+A,(y+A)-(d+A)]=A$. In other words,

$$
(y+A)-(d+A) \in \operatorname{Ann}_{L / A}^{\text {right }}(x+A)=S / A
$$

or

$$
y+A \in K / A+S / A .
$$

The fact that $y$ is an arbitrary element of $L$ shows that $L / A=K / A+S / A$. Furthermore, the choice of $B$ shows that $(S / A) \cap(K / A)=(S / A) \cap((B+A) / A)$ is zero. Thus $L / A=$ $K / A \oplus S / A$. An isomorphism

$$
S / A \cong(L / A) /(K / A) \cong L / K
$$

shows that $S$ / $A$ is a Lie algebra, having abelian subalgebra of dimension 4 . From the proof of Lemma 3 we can see that every cyclic subalgebra of $S / A$ is an ideal of $L / A$. Hence $L / A$ is a direct sum of two abelian ideals, so that $L / A$ is abelian. In particular, it is a Lie algebra. This means that $\operatorname{Leib}(L) \leqslant A$, and we obtain a contradiction. This contradiction shows that $K$ is $\langle x\rangle$-nilpotent for each element $x \in L$.

Let $x$ be an element of $L$ such that $x \notin \operatorname{Ann}_{L}(K)$. Then $\langle x, K\rangle$ is an ideal. Since $K$ is $\langle x\rangle$ nilpotent, $\langle x, K\rangle$ is nilpotent. In this case $\zeta(\langle x, K\rangle) \neq\langle 0\rangle$. Since $\langle x, K\rangle$ is an ideal of $L$, Lemma 4 implies that $\zeta(\langle x, K\rangle)$ is an ideal of $L$. The choice of $x$ implies that $Z_{1}=\zeta(\langle x, K\rangle) \neq K$. If 
we suppose that $K$ does not include $Z_{1}$, then $Z_{1}$ contains an element $\alpha x+b$ for some non-zero $\alpha \in F$. Then for arbitrary element $c \in K$ we obtain

$$
0=[\alpha x+b, c]=\alpha[x, c]+[b, c]=\alpha[x, c] .
$$

Since $\alpha \neq 0,[x, c]=0$. It is true for each element $c \in K$, so that $[x, K]=\langle 0\rangle$, and we obtain a contradiction with a choice of $x$. This contradiction shows that $K$ includes $Z_{1}$.

Suppose that $\operatorname{dim}_{F}\left(Z_{1}\right)=1$. If $y$ is an arbitrary element of $L$, then by above proved $\langle y, K\rangle$ is nilpotent. Since $Z_{1}$ is an ideal of $L$, and hence of $\langle y, K\rangle$, the intersection $\zeta(\langle y, K\rangle) \cap Z_{1}$ is non-zero [14, Lemma 2.4]. The fact that $\operatorname{dim}_{F}\left(Z_{1}\right)=1$ implies that $\zeta(\langle y, K\rangle) \cap Z_{1}=Z_{1}$. Since it is true for every element $y \in L$, we obtain that $Z_{1} \leqslant \zeta(L)$.

Suppose that $\operatorname{dim}_{F}\left(Z_{1}\right)=2$. If $L=\operatorname{Ann}_{L}\left(Z_{1}\right)$, then again $Z_{1} \leqslant \zeta(L)$. Therefore assume that $L \neq \operatorname{Ann}_{L}\left(Z_{1}\right)$ and choose an element $y$ such that $y \notin \operatorname{Ann}_{L}\left(Z_{1}\right)$. Then again $\langle y, K\rangle$ is nilpotent. Since $Z_{1}$ is an ideal of $L$, and hence of $\langle y, K\rangle$, the intersection $\zeta(\langle y, K\rangle) \cap Z_{1}=Z_{2}$ is non-zero [14, Lemma 2.4]. The choice of $y$ implies that $Z_{2} \neq Z_{1}$. Thus $\operatorname{dim}_{F}\left(Z_{2}\right)=1$. Using the above arguments, we obtain an inclusion $Z_{2} \leqslant \zeta(L)$. Hence in every case $\zeta(L) \cap K$ is non-zero. Repeating the above arguments we obtain that $K \leqslant \zeta_{3}(L)$. Since $L / K$ is abelian, it follows that $L$ is nilpotent.

Theorem 2. Let $L$ be a Leibniz algebra, having a dense family of ideals. Suppose that $L / L e i b(L)$ includes an abelian subalgebra of dimension 4 . Then either every subalgebra of $L$ is an ideal or $L$ satisfies the following conditions:

(a) $\operatorname{dim}_{F}(\operatorname{Leib}(L)) \leqslant 3 ;$

(b) L is nilpotent;

(c) $L /$ Leib $(L)$ is abelian;

(d) L includes an ideal $E$ such that $\operatorname{Leib}(L) \leqslant \zeta(E)$ and $[v, v] \neq 0$ for every element $v$ of $E$ such that $v \notin \operatorname{Leib}(L)$;

(e) if $\operatorname{dim}_{F}(\operatorname{Leib}(L))=3$, then $\operatorname{dim}_{F}(L / E) \leqslant 3$;

(f) if $\operatorname{dim}_{F}(\operatorname{Leib}(L))=2$, then $\operatorname{dim}_{F}(L / E) \leqslant 6$;

(g) if $\operatorname{dim}_{F}(\operatorname{Leib}(L))=1$, then $\operatorname{dim}_{F}(L / E) \leqslant 8$.

In particular, if $\operatorname{dim}_{F}(\operatorname{Leib}(L))=1$, then every subalgebra of $E$ is an ideal of $L$.

Proof. Put $K=\operatorname{Leib}(L)$. If $L$ includes an abelian subalgebra of dimension 4 , then Theorem 1 shows that every subalgebra of $L$ is an ideal. Suppose that $L$ has the subalgebras, which are not ideals. Then $\operatorname{dim}_{F}(K) \leqslant 3$. By Lemma $5 L / K$ is abelian and $L$ is nilpotent.

Suppose that $\operatorname{dim}_{F}(K)=3$. A factor-algebra $L / \operatorname{Ann}_{L}^{\text {left }}(K)$ is isomorphic to some subalgebra of algebra of derivations of $K$ [11, Proposition 3.2]. Moreover, the fact that $L$ is nilpotent implies that $L / \operatorname{Ann}_{L}^{\text {left }}(K)$ is isomorphic to some subalgebra of all zero-triangular matrices $N T_{3}(F)$. In particular, we obtain that

$$
\operatorname{dim}_{F}\left(L / \operatorname{Ann}_{L}^{\text {left }}(K)\right) \leqslant 3
$$


We note that $\operatorname{Ann}_{L}^{\text {left }}(K)=\operatorname{Ann}_{L}(K)$. Put $E=\operatorname{Ann}_{L}(K)$. Assume that $E$ has an element $x \notin K$ such that $[x, x]=0$. Since $L / K$ is abelian, $\langle x, K\rangle$ is an ideal of $L$. The choice of element $x$ follows that this subalgebra is abelian. Since $\operatorname{dim}_{F}(\langle x, K\rangle)=4$, Theorem 1 implies that every subalgebra of $L$ is an ideal, and we obtain a contradiction. This contradiction shows that $[x, x] \neq 0$ for every $x$ of $E$ such that $x \notin K$.

Suppose that $\operatorname{dim}_{F}(K)=2$. In this case $L / \operatorname{Ann}_{L}^{\text {left }}(K)$ is isomorphic to some subalgebra of all triangular matrices $T_{2}(F)$. In particular,

$$
\operatorname{dim}_{F}\left(L / \operatorname{Ann}_{L}^{l e f t}(K)\right) \leqslant 1
$$

Put $D=\operatorname{Ann}_{L}(K)$. Assume that $D$ has an element $x \notin K$ such that $[x, x]=0$. Then $\langle x, K\rangle$ is abelian and has dimension 3 . Since $L / K$ is abelian, $\langle x, K\rangle$ is an ideal of $L$. For arbitrary element $y \in D$ consider the mappings

$$
1_{y}:\langle x, K\rangle \rightarrow\langle x, K\rangle
$$

and

$$
\mathrm{r}_{y}:\langle x, K\rangle \rightarrow\langle x, K\rangle,
$$

defined by the rule: $1_{y}(b)=[y, b]$ and $\mathrm{r}_{y}(b)=[b, y], b \in\langle x, K\rangle$. If $\left\{a_{1}, a_{2}\right\}$ is the basis of $K$, then the matrix of $l_{y}$ in a basis $\left\{a_{1}, a_{2}, x\right\}$ has a following form

$$
\left(\begin{array}{lll}
0 & 0 & 0 \\
0 & 0 & \alpha \\
0 & 0 & \beta
\end{array}\right)
$$

for some $\alpha, \beta \in F$. It follows that $\operatorname{dim}_{F}\left(D / \operatorname{Ann}_{D}^{\text {left }}(\langle x, K\rangle)\right) \leqslant 2$. Further, the matrix of $\mathrm{r}_{y}$ in a basis $\left\{a_{1}, a_{2}, x\right\}$ also has a following form

$$
\left(\begin{array}{lll}
0 & 0 & 0 \\
0 & 0 & \lambda \\
0 & 0 & \mu
\end{array}\right)
$$

for some $\lambda, \mu \in F$. It follows that $D / \operatorname{Ann}_{D}^{\text {right }}(\langle x, K\rangle)$ has a dimension at most 2. Put

$$
B=\operatorname{Ann}_{D}^{\text {left }}(\langle x, K\rangle) \cap \operatorname{Ann}_{D}^{\text {right }}(\langle x, K\rangle)=\operatorname{Ann}_{D}(\langle x, K\rangle) .
$$

Since $D$ is an ideal of $L, B$ is an ideal of $L$. The choice of $B$ implies that

$$
\operatorname{dim}_{F}(L / B) \leqslant 1+2+2=5 .
$$

Assume that $B$ has an element $u \notin\langle x, K\rangle$ such that $[u, u]=0$. The choice of this element yields that the subalgebra $\langle u, x, K\rangle$ is abelian. Since $\operatorname{dim}_{F}(\langle u, x, K\rangle)=4$, Theorem 1 implies that every subalgebra of $L$ is an ideal, and we obtain a contradiction. This contradiction shows that $[u, u] \neq 0$ for every $u$ of $B$ such that $u \notin\langle x, K\rangle$. Since $L / K$ is abelian, $\langle x, K\rangle / K$ has a complement $E / K$ in $B / K$ :

$$
B / K=\langle x, K\rangle / K \oplus E / K \text {. }
$$

Moreover, $E$ is an ideal of $L$. Since $x \notin K,\langle x, K\rangle=K \oplus F x$. The fact that $[x, x]=0$ implies that $\langle x\rangle=F x$, and we have $B=E \oplus\langle x\rangle$. Then $\operatorname{dim}_{F}(E)=\operatorname{dim}_{F}(B)-1$, and therefore 
$\operatorname{dim}_{F}(L / E) \leqslant 5+1=6$. The choice of $E$ implies that $[v, v] \neq 0$ for every $v$ of $E$ such that $v \notin K$.

Finally, suppose that $\operatorname{dim}_{F}(K)=1$. Using the above arguments, in this case we obtain that $L$ includes an ideal $E \geqslant K$ such that $\operatorname{dim}_{F}(L / E) \leqslant 8$ and $[v, v] \neq 0$ for every $v$ of $E$ such that $v \notin K$.

In Theorem 2 appeared the following type of Leibniz algebras: $\operatorname{dim}_{F}(\operatorname{Leib}(L)) \leqslant 3$, $\operatorname{Leib}(L) \leqslant \zeta(L)$ and $[x, x] \neq 0$ for every $x \notin \operatorname{Leib}(L)$. If $\operatorname{dim}_{F}(\operatorname{Leib}(L))=1$, then every subalgebra of $L$ is an ideal. But the cases when $\operatorname{dim}_{F}(\operatorname{Leib}(L))=2$ or 3 , require the separate consideration.

If in Theorem $2 \operatorname{Leib}(L)=\operatorname{Ann}_{L}(\operatorname{Leib}(L))$, then we obtain the algebras of dimension 6, 8, 9 respectively. These cases also require the separate consideration.

Finally, let $L$ be a Lie algebra, having dense family of ideals, and suppose that every abelian subalgebra of $L$ has dimension at most 3 . If $L$ includes an abelian subalgebra $A$ of dimension 3 , then by Lemma $1 A$ is an ideal of $L$. Then $A=\operatorname{Ann}_{L}(A)$, and hence $L / \operatorname{Ann}_{L}(A)$ has dimension at most $9, L$ has a dimension at most 12 . Thus we can see that the case, when a Leibniz algebra, having dense family of ideals, has a dimension at most 15 , require the separate consideration.

\section{REFERENCES}

[1] Barnes D. Schunck classes of soluble Leibniz algebras. Comm. Algebra 2013, 41 (11), 4046-4065. doi:10.1080/00927872.2012.700978

[2] Blokh A. On a generalization of the concept of Lie algebra. Dokl. Akad. Nauk SSSR 1965, 165 (3), 471-473.

[3] Chernikov S.N. Groups with a dense system of complemented subgroups. In "Some Questions in Group Theory", Ins. Mat. Akad. Nauk Ukr. SSR, Kiev, 1975, 5-29.

[4] Chupordia V.A., Kurdachenko L.A., Subbotin I. Ya. On some "minimal" Leibniz algebras. J. Algebra Appl. 2017, 16 (05), 1750082. doi:10.1142/S0219498817500827

[5] Chupordia V.A., Pypka A.A., Semko N.N., Yashchuk V.S. Leibniz algebras: a brief review of current results. Carpathian Math. Publ. 2019, 11 (2), 250-257. doi:10.15330/cmp.11.2.250-257

[6] Kirichenko V.V., Kurdachenko L.A., Pypka A.A., Subbotin I.Ya. Some aspects of Leibniz algebra theory. Algebra Discrete Math. 2017, 24 (1), 1-33.

[7] Kurdachenko L.A., Goretskii V.E. Groups with a complete system of almost-normal subgroups. Ukrainian Math. J. 1983, 35, 37-40. doi:10.1007/BF01093160

[8] Kurdachenko L.A., Kuzennyi N.F., Pylaev V.V. Infinite groups with a generalized dense system of subnormal subgroups. Ukrainian Math. J. 1981, 33, 313-316. doi:10.1007/BF01085574

[9] Kurdachenko L.A, Kuzennyi N.F., Semko N.N. Groups with dense systems of infinite subgroups. Dokl. Akad. Nauk Ukr. SSR. Ser. A. 1985, 3, 7-9.

[10] Kurdachenko L.A, Kuzennyi N.F., Semko N.N. Groups with a dense system of infinite almost normal subgroups. Ukrainian Math. J. 1991, 43, 904-908. doi:10.1007/BF01058691

[11] Kurdachenko L.A., Otal J., Pypka A.A. Relationships between factors of canonical central series of Leibniz algebras. Eur. J. Math. 2016, 2 (2), 565-577. doi:10.1007/s40879-016-0093-5

[12] Kurdachenko L.A., Otal J., Subbotin I.Ya. On some properties of the upper central series in Leibniz algebras. Comment. Math. Univ. Carolin. 2019, 60 (2), 161-175.

[13] Kurdachenko L.A., Semko N.N., Subbotin I.Ya. The Leibniz algebras whose subalgebras are ideals. Open Math. 2017, 15 (1), 92-100. doi:10.1515/math-2017-0010 
[14] Kurdachenko L.A., Subbotin I.Y., Semko N.N. From groups to Leibniz algebras: common approaches, parallel results. Adv. Group Theory Appl. 2018, 5, 1-31. doi:10.4399/97888255161421

[15] Kurdachenko L.A., Subbotin I.Ya., Yashchuk V.S. Leibniz Algebras whose subideals are ideals. J. Algebra Appl. 2018, 17 (08) 1850151. doi:10.1142/S0219498818501517

[16] Loday J.L. Une version non commutative des algèbres de Lie: les algèbras de Leibniz. Enseign. Math. 1993, 39, 269-293.

[17] Mann A. Groups with dense normal subgroups. Israel J. Math. 1968, 6, 13-25. doi:10.1007/BF02771600

[18] Semko N.N. Structure of locally graded nonnilpotent CDN[ ]-groups. Ukrainian Math. J. 1997, 49, $883-893$. doi:10.1007/BF02513428

[19] Semko N.N. Structure of one class of groups with conditions of denseness of normality for subgroups. Ukrainian Math. J. 1997, 49, 1292-1295. doi:10.1007/BF02487555

[20] Semko N.N. On the construction of CDN[ ]-groups with elementary commutant of rank two. Ukrainian Math. J. 1997, 49, 1570-1577. doi:10.1007/BF02487442

[21] Semko N.N. On the structure of CDN[ ]-groups. Ukrainian Math. J. 1998, 50, 1431-1441. doi:10.1007/ BF02525249

[22] Semko N.N. Structure of locally graded CDN( ]-groups. Ukrainian Math. J. 1998, 50, 1750-1754. doi:10.1007/BF02524481

[23] Semko N.N. Structure of locally graded CDN[ )-groups. Ukrainian Math. J. 1999, 51, 427-433. doi:10.1007/BF02592479

[24] Semko N.N., Skaskiv L.V., Yarovaya O.A. Linear groups saturated by subgroups of finite central dimension. Algebra Discrete Math. 2020, 29 (1), 117-128. doi:10.12958/adm1317

Received 02.05.2020

Семко М.М., Скасків А.В., Ярова О.А. Алгебри Аейбніца з шільним сімейством ідеалів // Карпатські матем. публ. - 2020. - Т.12, №2. - С. 451-460.

Кажуть, що алгебра $\Lambda$ ейбніца $L$ має шільне сімейство ідеалів, якщо для кожної пари таких підалгебр $A, B$ з $L$, що $A \leqslant B$ та $A$ не є максимальною в $B$, існує такий ідеал $S$, що $A \leqslant S \leqslant B$. У статті досліджуються алгебри Аейбніца з щільним сімейством ідеалів.

Ключові слова і фрази: алгебра $е$ еибніца, алгебра $\Lambda$ i, ідеал, щільне сімейство, нільпотентна

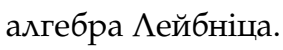

